\title{
PRESERVAÇÃO DA ARCADA VASCULAR DO CÓLON DIREITO PARA O ALONGAMENTO MESENTERIAL NA CIRURGIA DO RESERVATÓRIO ILEAL
}

\author{
PRESERVATION OF THE VASCULAR ARCADE OF THE RIGHT COLON \\ TO THE MESENTERY LENGTHENING IN THE ILEAL POUCH PROCEDURE
}

\author{
Juvenal Ricardo Navarro Góes, TCBC-SP ${ }^{2}$ \\ João José Fagundes, TCBC-SP ${ }^{3}$ \\ Cláudio Saddy Rodrigues $\mathrm{Coy}^{3}$ \\ Maria de Lourdes Setsuko Ayrizono ${ }^{4}$ \\ Luis Sérgio Leonardi, TCBC-SP ${ }^{1}$
}

\begin{abstract}
RESUMO: Objetivo: Avaliar a eficácia da preservação da arcada vascular do cólon direito (AVCD) na manutenção da perfusão sangüínea do reservatório após manobras de alongamento do mesentério ileal. Métodos: O estudo incluiu 46 pacientes (janeiro/1990 a julho/2000, para o tratamento de retocolite ulcerativa (RCU) e polipose adenomatosa familiar (PAF), que foram divididos em dois grupos: grupo com preservação da arcada (CPA), 27 pacientes, média de idade - 34,0 (19-53) anos, 15 (55,6\%) eram do sexo feminino e 24 (88,9\%) eram brancos; e grupo sem preservação da arcada (SPA), 19 pacientes, média de idade 41,5 (13 - 62) anos, oito $(42,1 \%)$ eram do sexo feminino e $18(94,8 \%)$ eram brancos. Trinta pacientes $(65,2 \%)$ apresentavam RCU e 16 (34,7\%) PAF. Foram comparadas a ocorrência de complicações atribuídas à má perfusão sangüínea ou tensão na anastomose ileoanal, assim como as indicações de reoperações. Resultados: No grupo CPA, em quatro pacientes $(18,5 \%)$, as complicações estavam relacionadas ao comprometimento da viabilidade intestinal ou à tensão ao nível da anastomose. No grupo SPA, estas complicações ocorreram em sete pacientes $(36,9 \%)$. Foram reoperados três pacientes $(11,1 \%)$ do grupo CPA, por causas relacionadas ou à má perfusão sangüínea ou à tensão excessiva no nível da anastomose e no grupo SPA cinco (26,3\%), pela mesma causa. Conclusões: Apesar das limitações metodológicas, observou-se tendência para menor ocorrência de complicações isquêmicas no grupo com preservação da AVCD. Quando o abaixamento do reservatório ileal tem que ser ao nível da linha pectínea, torna-se importante a perfusão sangüínea via AVCD como auxiliar na preservação de sua viabilidade.
\end{abstract}

Descritores: Retocolite ulcerativa; Polipose adenomatosa familiar; Bolsa ileal; Reservatório ileal pélvico; Anastomose ileoanal.

\section{INTRODUÇÃO}

A retocolectomia total, com anastomose ileoanal utilizando-se reservatório ileal (RTRI), para o tratamento da retocolite ulcerativa (RCU) e a polipose adenomatosa familiar (PAF), representou o grande avanço técnico na cirurgia colorretal no século XX. A evolução dos conceitos relativos ao tratamento cirúrgico dessas doenças norteouse, basicamente, pela necessidade de ressecção de toda mucosa doente e preservação da continência ${ }^{1,2}$. No Brasil, a experiência tem também refletido essa evolução $0^{3-15}$. Alguns detalhes técnicos desse procedimento, como a mobi-

1. Professor Titular.

2. Professor Associado, Coordenador do Grupo de Coloproctologia.

3. Professor Doutor.

4. Mestre em Cirurgia. Pós-graduanda do Grupo de Coloproctologia.

Recebido em 18/10/2001

Aceito para publicação em 26/12/2001

Trabalho realizado no Departamento de Cirurgia, Disciplina de Moléstias do Aparelho Digestivo, Grupo de Coloproctologia, da Faculdade de Ciências Médicas da UNICAMP 
lização do íleo terminal, as ligaduras de vasos mesenteriais e a preparação de arcadas vasculares, são importantes para o abaixamento bem-sucedido do reservatório até o canal anal, com anastomose ao nível da linha pectínea, livre de tensão e com viabilidade assegurada. Situação diferente é a utilização do duplo grampeamento. Trata-se de anastomose realizada no topo do canal anal, com menor ocorrência de comprometimento da viabilidade ou outras complicações, em razão do menor alongamento mesentérico necessário.

Alguns estudos têm mostrado a preocupação com o alongamento mesenterial e o desenvolvimento de alternativas técnicas, visando à mobilização mais ampla possível do mesentério ileal, tentando evitar-se com isso o abandono da cirurgia ou, pelo menos, a diminuição da ocorrência de complicações como isquemia, deiscência e retração de reservatório e estenoses anastomóticas ${ }^{16-25}$.

O objetivo deste estudo foi, pela análise das complicações pós-operatórias, avaliar a eficácia da preservação da arcada vascular do cólon direito (AVCD), como garantia da perfusão sangüínea do reservatório ileal, complementar àquela proveniente dos ramos ileais da artéria mesentérica superior (AMS). Este estudo foi aprovado pelo Comitê de Ética em Pesquisa desta instituição.

\section{MÉTODOS}

O presente estudo analisa a técnica de preservação da AVCD, desenvolvida no Grupo de Coloproctologia da Disciplina de Moléstias do Aparelho Digestivo da Faculdade de Ciências Médicas da UNICAMP, em pacientes que foram submetidos à RTRI, para o tratamento de RCU e PAF, de janeiro de 1990 a julho de 2000 e constou de 46 pacientes. Foram estudados dois grupos: grupo com preservação da arcada (CPA), 27 pacientes, média de idade $34,0(19-53)$ anos, sendo $15(55,6 \%)$ do sexo feminino e $24(88,9 \%)$ pertencendo à raça branca; e grupo sem preservação da arcada (SPA), 19 pacientes, média de idade 41,5 $(13-62)$ anos, sendo $11(57,8 \%)$ do sexo masculino e 18 $(94,8 \%)$ pertencendo à raça branca. Trinta pacientes $(65,2 \%)$ eram portadores de RCU e 16 (34,7\%) de PAF.

Reservatórios em forma de ' $\mathrm{S}$ ' foram utilizados em sete pacientes $(15,2 \%)$, em ' $\mathrm{J}$ ' em $23(50,0 \%)$ e em forma mista ('dupla-câmara') $)^{9}$ em $16(34,7 \%)$. Todos os pacientes tiveram ileostomia derivativa em alça.

Para o Grupo CPA, a colectomia total iniciou-se, sistematicamente, pelo lado direito dos cólons. Na liberação cólica e do íleo terminal procedeu-se de modo a expor toda a extensão da vascularização do cólon direito, desde a artéria cólica média até o mesentério do íleo terminal. Passo seguinte, procedeu-se à ligadura dos vasos retos emergentes da AVCD, no plano entre esta e a parede cólica, de modo a preservar a integridade da arcada, mantendo a comunicação do ramo direito da artéria cólica média com a vascularização do mesentério do íleo terminal. Em seguida, determinou-se o ponto considerado ápice do reservatório e que deveria ultrapassar a sínfise púbica em pelo menos seis centímetros. Procedia-se à ligadura dos ramos mesentéricos principais, à medida que se constata- va que estavam sob tensão, podendo, eventualmente, incluir-se ligaduras de ramos secundários do mesentério do íleo terminal, desde que assegurada a perfusão sanguiínea e como já anteriormente descrito ${ }^{21,22,24}$ (Figuras 1 e 2).

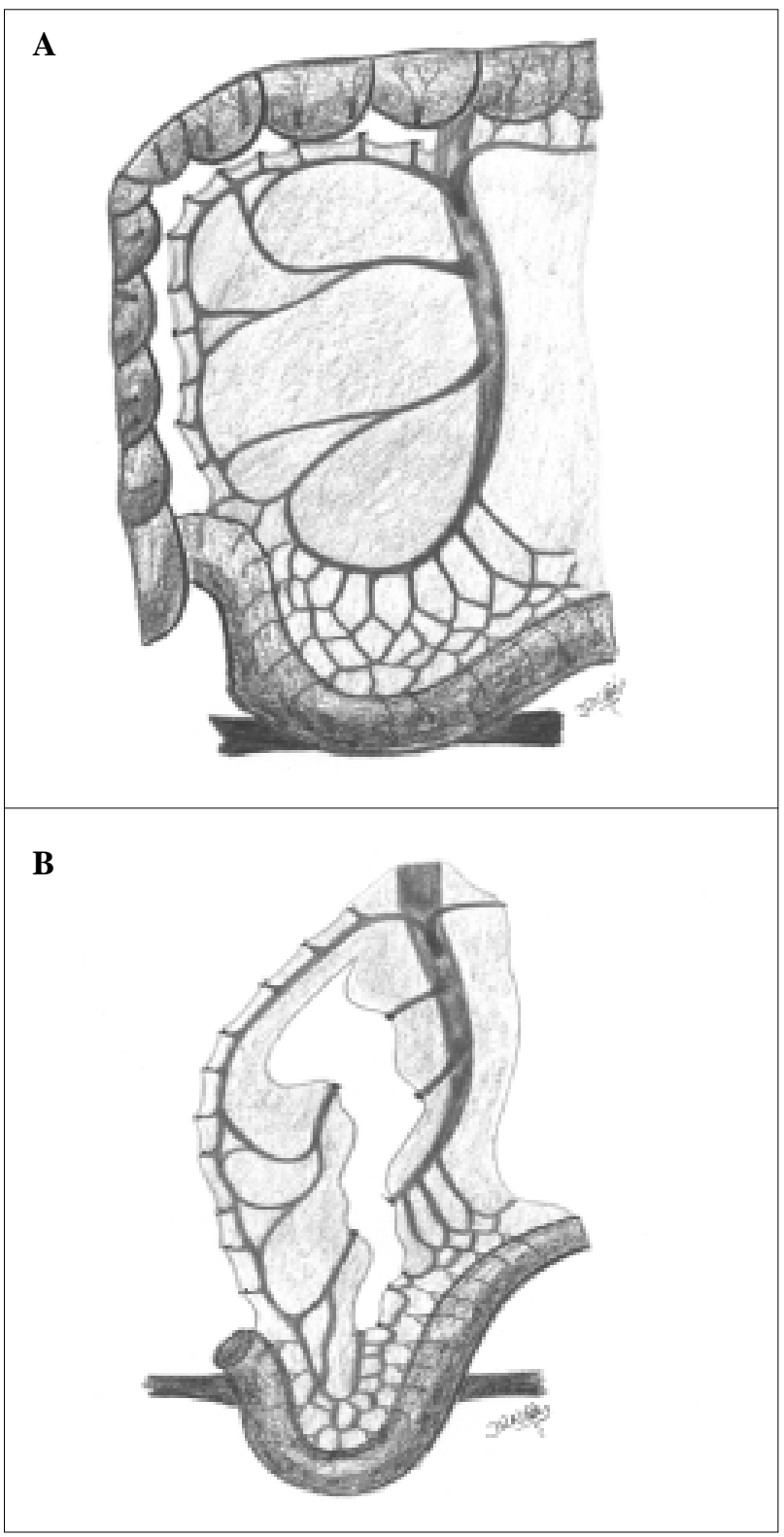

Figura 1 - A) Colectomia realizada com a preservação da AVCD; B) Alongamento mesentérico com ligadura dos vasos mesenteriais.

$\mathrm{O}$ reservatório era abaixado à pelve e anastomosado ao canal anal por via endoanal, com pontos separados de ácido poliglicólico 3-0, gastrointestinal (Vicryl ${ }^{\circledR}$, Ethicon), interessando o epitélio e musculatura esfincteriana ao nível da linha pectínea e toda a espessura da parede da alça ileal. 

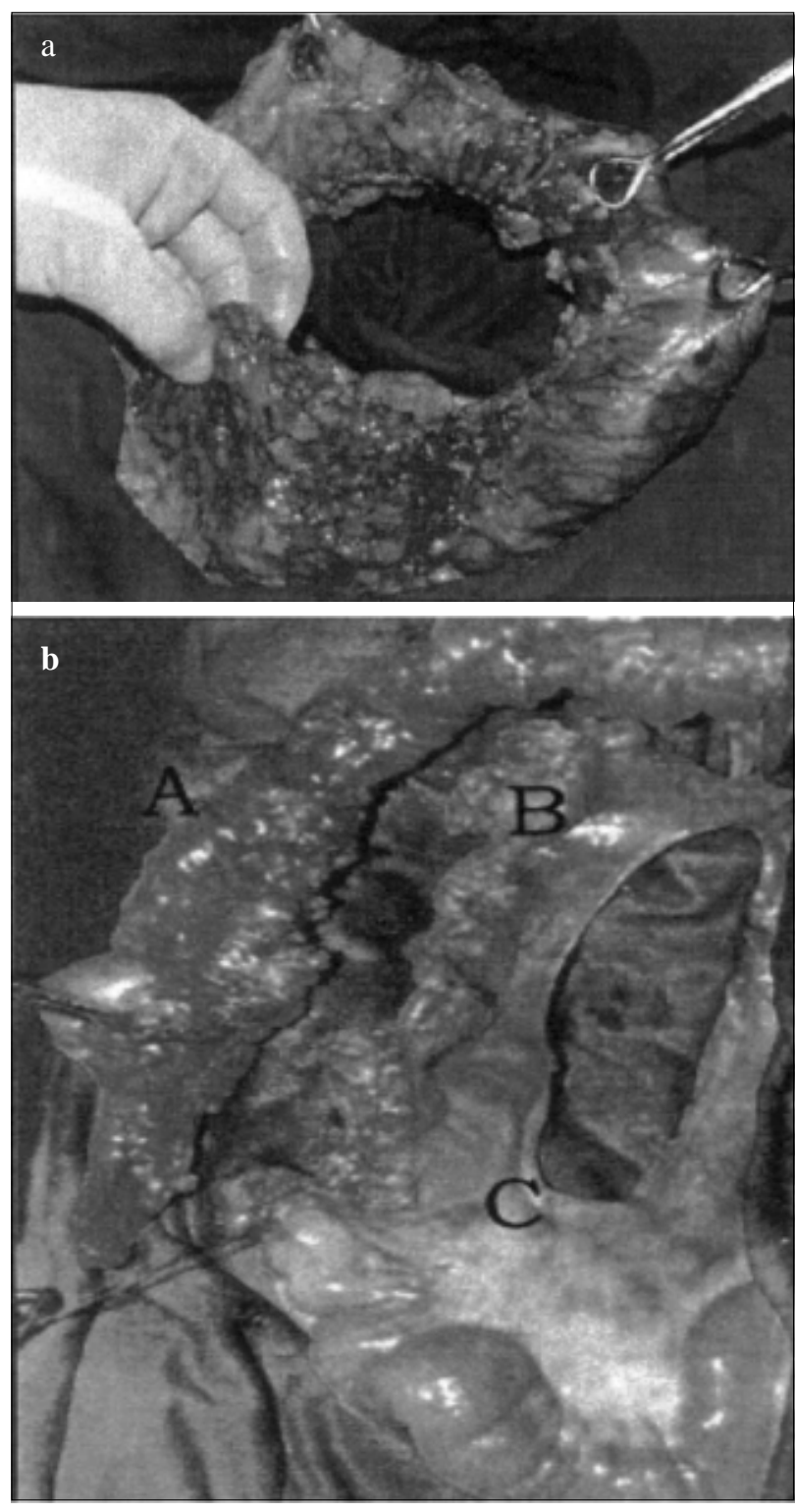

Figura 2-a) Arcada vascular do cólon direito preservada; b) A. cólon ressecado, B. arcada preservada, $C$. mesentério ileal.

Para o Grupo SPA, o procedimento foi semelhante, exceto pelo nível em que se procedeu às ligaduras dos vasos mesentéricos, a saber: artéria ileocecal, artéria cólica direita, artéria cólica média e demais vasos dos segmentos cólicos subseqüientes.

Foram comparadas as complicações atribuídas à ocorrência de má perfusão sangüínea ao reservatório ileal, ou, então, à ocorrência de tensão na anastomose ileoanal, entre os grupos CPA e SPA. Foram também comparadas as indicações de reoperações para o tratamento dessas complicações.

$\mathrm{O}$ estudo não se baseou em amostras aleatórias e prospectivas, além de serem populações de pequeno tamanho em cada grupo, o que obviamente limita o poder de se estabelecerem conclusões com significância estatís- tica. Foi feita apenas estimativa comparativa de tendências, nas duas séries históricas, baseada na projeção de crescimento constante do tamanho da amostra, mantendo as proporções atuais de ocorrência de complicações e necessidade de reoperações.

\section{RESULTADOS}

Dos 27 pacientes que foram submetidos à RTRI, com preservação da AVCD (grupo CPA), 17 (62,9\%) não apresentaram complicações pós-operatórias. Dos dez pacientes $(37,0 \%)$ com complicações, em cinco $(18,5 \%)$ estas estavam relacionadas ao comprometimento da viabilidade intestinal ou à tensão no nível da anastomose reservatório-canal anal.

Dos 19 pacientes submetidos ao procedimento mas sem preservação da AVCD (grupo SPA), sete $(36,9 \%)$ não apresentaram nenhum tipo de complicações. Dos 12 pacientes $(63,1 \%)$ com complicações, sete $(36,9 \%)$ tiveram complicações relacionadas ao comprometimento da viabilidade intestinal ou à tensão no nível da anastomose. Esses resultados estão resumidos na Tabela 1.

Isquemia do reservatório ocorreu em dois pacientes, sendo um $(3,7 \%)$ do grupo CPA e um $(5,2 \%)$ do grupo SPA. O caso do grupo CPA foi reoperado no quarto dia PO, desfeita a anastomose reservatório-canal anal, ressecado o reservatório e instalada ileostomia terminal. Apresentou aspiração pulmonar na indução anestésica desta reoperação e, após várias complicações gerais, evoluiu para o óbito, no $45^{\circ}$ dia $\mathrm{PO}$. O paciente do grupo SPA foi reoperado no terceiro dia PO, desfeita a anastomose com exteriorização do remanescente do reservatório. Este paciente teve seu reservatório refeito e ressuturado ao canal anal 47 meses mais tarde, tendo apresentado ótima evolução.

Comprometimento parcial da viabilidade do reservatório foi observado em quatro pacientes, sendo um $(3,7 \%)$ do grupo CPA e três $(15,7 \%)$ do grupo SPA. Para o paciente do grupo CPA foi possível manter-se o reservatório in loco sendo posteriormente submetido à laparotomia exploradora utilizando-se dilatações intra-operatórias com balão introduzido via anal, apresentando boa evolução. No grupo SPA, um paciente não teve sucesso com as dilatações realizadas, permanecendo por dois anos com ileostomia. Foi, posteriormente, submetido à excisão do reservatório, dilatações do canal anal, confecção de novo reservatório e reanastomose ao canal anal. Teve boa evolução pós-operatória, a ileostomia foi fechada, mas, tardiamente, constatou-se a presença de fístula reservatório-vesical. A ileostomia foi novamente instalada, corrigida a fístula reservatório-vesical e o paciente permanece derivado. Os dois outros pacientes tiveram apenas necrose parcial de mucosa e evoluíram bem com dilatações realizadas ambulatorialmente.

Comprometimento da viabilidade da alça aferente ao reservatório, decorrência de ligaduras vasculares complementares no mesentério ileal, durante manobras de alongamento, foi observado em um paciente $(3,7 \%)$ do grupo CPA, que evoluiu com perfuração do segmento afeta- 
Tabela 1

Ocorrência de complicações pós-operatórias e aquelas decorrentes do comprometimento da perfusão sangüínea ao reservatório ou pela tensão excessiva no nível da anastomose reservatório-anal, nos grupos com e sem preservação da AVCD

\begin{tabular}{l|c|c|c}
\hline \multirow{2}{*}{ Grupos } & \multicolumn{3}{|c}{ Complicações } \\
\cline { 2 - 4 } CPA (27 pacientes) & Não & Sim & Isquemia/Tensão \\
SPA (19 pacientes) & $17(62,9 \%)$ & $10(37,0 \%)$ & $5(18,5 \%)$ \\
\hline
\end{tabular}

$C P A=$ Com preservação de arcada

$S P A=$ Sem preservação de arcada

do, formação de abscesso e fistulização enterocutânea. A cura da fístula deu-se em sete dias, mas evoluiu tardiamente para estenose. Após o quarto mês PO, e garantido pela circulação da AVCD preservada, foi possível a ressecção segura do segmento afetado, sem comprometimento do reservatório abaixado.

Restrição volumétrica com diminuição da complacência do reservatório ocorreu em conseqüência do comprometimento de sua perfusão sangüínea e foi verificada em dois pacientes, sendo um $(3,7 \%)$ do grupo CPA e um $(5,2 \%)$ do grupo SPA. Deiscência da anastomose ocorreu em três pacientes, sendo um (3,7\%) do grupo CPA e dois $(10,5 \%)$ do grupo SPA. Em todos, a deiscência aconteceu de forma parcial, sem que a retração do reservatório tenha ocorrido.

Estenose da anastomose reservatório-canal anal foi observada em dois pacientes $(10,5 \%)$, ambos do grupo SPA, tendo sido atribuída à má perfusão sangüínea ao reservatório, sendo em um deles com comprometimento apenas distal e no outro apenas da camada mucosa.
Apesar de complicação rara, a síndrome da artéria mesentérica superior ocorreu em um paciente desta série $(5,2 \%)$, pertencente ao grupo SPA, obrigando à reoperação. As complicações acima descritas estão resumidas na Tabela 2.

Dentre os 27 pacientes pertencentes ao grupo CPA, dez $(37,0 \%)$ tiveram que ser reoperados, e destes, três $(11,1 \%)$ por causas relacionadas ou à má perfusão sangüínea ou à tensão excessiva no nível da anastomose. No grupo SPA, dos 19 pacientes operados, $12(63,1 \%)$ foram reoperados e, destes, cinco $(26,3 \%)$ tiveram como causa complicações relacionadas ou à má perfusão sangüínea ou à tensão excessiva no nível da anastomose.

Não houve, portanto, até o momento, diferença com significância estatística quanto à ocorrência de complicações isquêmicas ou decorrentes de tensão excessiva no nível da anastomose e a necessidade de reoperações entre os dois grupos, mas, estimando-se a tendência dessas intercorrências, mantidas as proporções atuais de crescimento da amostra e a taxa de suas ocorrências, é possível identificar-se menor probabilidade para o grupo CPA.

Tabela 2

Distribuição das complicações decorrentes do comprometimento da perfusão sangüínea ao reservatório ou pela tensão excessiva na anastomose reservatório-anal, nos grupos com e sem preservação da arcada vascular marginal do cólon direito.

\begin{tabular}{|c|c|c|c|c|}
\hline \multirow[b]{3}{*}{ Complicações } & \multicolumn{4}{|c|}{ Grupo } \\
\hline & \multicolumn{2}{|c|}{$C P A$} & \multicolumn{2}{|c|}{$S P A$} \\
\hline & $N$ & $(\%)$ & $N$ & $(\%)$ \\
\hline Isquemia do reservatório & 1 & $(3,7)$ & 1 & $(5,2)$ \\
\hline Comprometimento parcial da viabilidade do reservatório & 1 & $(3,7)$ & 3 & $(15,7)$ \\
\hline Comprometimento da viabilidade da alça aferente & 1 & $(3,7)$ & 0 & \\
\hline Restrição volumétrica ou da complacência do reservatório & 1 & $(3,7)$ & 1 & $(5,2)$ \\
\hline Deiscência da anastomose reservatório — anal & 1 & $(3,7)$ & 2 & $(10,5)$ \\
\hline Estenose da anastomose reservatório — anal & 0 & & 2 & $(10,5)$ \\
\hline Estenose da alça aferente & 1 & $(3,7)$ & 0 & \\
\hline Síndrome da artéria mesentérica superior & 0 & & 1 & $(5,2)$ \\
\hline Total & 6 & (5 pac.) & 10 & (7 pac.) \\
\hline
\end{tabular}

CPA - Com preservação da arcada

SPA - Sem preservação da arcada 


\section{DISCUSSÃO}

O princípio do tratamento cirúrgico da $\mathrm{RCU}$ e da PAF está fundamentado no conceito de que a doença compromete a mucosa colorretal e que, portanto, esta deva ser ressecada totalmente, desde a linha pectínea, para que a cura seja plenamente conseguida. Este tipo de abordagem torna-se mandatória naqueles casos em que já existem displasia de alto grau, câncer em segmentos mais proximais, ou comprometimento da mucosa de canal anal por pólipos adenomatosos.

Conceitualmente, dois procedimentos cirúrgicos distintos podem ser utilizados para o tratamento da RCU e PAF com envolvimento retal importante. Um deles quando se utiliza a técnica do duplo grampeamento, apoiando-se no pressuposto de que a presença de mucosa anorretal residual não acarretará maiores preocupações para o pós-operatório tardio. O outro, quando é praticada anastomose manual por via endoanal, ao nível da linha pectínea, em decorrência da necessidade de ressecção de toda mucosa anorretal. O primeiro traduz abordagem por via anterior, com maior facilidade de execução. Já o segundo é cirurgia de abaixamento, o que implica procedimento mais difícil, necessidade de mais ampla mobilização do íleo terminal e divisões de vasos mesenteriais sob tensão. A este procedimento estará, logicamente, associada maior ocorrência de complicações e mesmo maior possibilidade de abandono da técnica caso o reservatório não alcance a linha pectínea, preservando sua viabilidade e livre de tensão.

A técnica de anastomose do reservatório ileal com o canal anal pelo duplo grampeamento, proposta desde a década de $80^{26-29}$, tem sido associada à melhor função ${ }^{30}$, mas é importante ressaltar que este fato pode ter a ver, entre outros fatores, com a menor extensão de ressecção de mucosa e, principalmente, de esfíncter anal interno, que se verifica quando da colocação alta, inadvertida ou intencional, do grampeador. Para cumprir-se a finalidade do tratamento cirúrgico da RCU e da PAF, a remoção da mucosa doente deveria ser a mais completa possível, evitando-se a construção de anastomoses distantes da linha pectínea, isto é, anastomose ileorretal baixa em vez de ileoanal. Trata-se, contudo, de procedimento nem sempre fácil, seja pela estreiteza da pelve, seja pela sua profundidade, o que pode acarretar indesejável tensão na anastomose ou alguns centímetros de mucosa remanescente.

Como enfatizado desde as experiências pioneiras com esse procedimento ${ }^{16,31}$, para o alcance sem tensão é freqüente a necessidade de realizar-se manobras de alongamento de mesentério, procedendo-se a secções de vasos mesenteriais, visando ao ganho, não raramente, de apenas um ou dois centímetros. Esse passo técnico deve ser balizado pela segurança, uma vez que o simples rompimento de um vaso na arcada secundária do mesentério pode significar o comprometimento definitivo da viabilidade distal do íleo terminal.

Em face dessas constatações, no tratamento de paciente que necessite da remoção de toda mucosa doente é importante que o cirurgião colorretal não ignore alterna- tivas técnicas para proceder à ressecção completa do reto, com a ablação da mucosa até a linha pectínea, suturando o reservatório ileal ao nível do canal anal, sem risco de isquemia ou tensão na sutura. Há que se ponderar entre o risco de se deixar mucosa residual doente no canal anal versus risco de perda do reservatório por isquemia secundária às manobras de alongamento.

Surpreendentemente, apesar das manobras de alongamento mesenterial serem discutidas na literatura, poucas são as referências quanto à ocorrência desse comprometimento da perfusão ao reservatório, de sua importância na determinação da morbidade, freqüência de perda do reservatório e nos resultados funcionais tardios ${ }^{30,32,33}$. A mobilização ileal à custa da preservação da AVCD e ligaduras dos vasos mesentéricos propiciou alongamentos bastante adequados, em acordo com estudos que foram realizados abordando esse aspecto da técnica ${ }^{17-19}$. Burnstein et $a l .{ }^{18}$, que realizavam o alongamento mesenterial à custa, principalmente, de ligaduras de vasos secundários do sistema formado pelos vasos ileocecais e mesentérica superior, preservando os troncos principais, observaram ser necessário que o ápice do reservatório alcançasse pelo menos $6 \mathrm{~cm}$ abaixo da sínfise púbica para que o abaixamento do reservatório até o canal anal se desse sem problemas.

Apesar da análise desta série de pacientes não permitir, até o momento, o estabelecimento de conclusões estatisticamente significantes, por não se tratar de estudo randomizado e prospectivo, foi possível identificar tendência para a menor ocorrência de complicações decorrentes da má perfusão dos reservatórios em seguida à utilização de manobras de alongamento mesenterial, assim como a necessidade de reoperações, nos pacientes em que houve a preservação da AVCD.

O exame detalhado das condições anatômicas de vascularização, comprimento e espessura de mesentério da região ileocólica deve ser feito antes de se iniciar a colectomia total, uma vez que, em situações especiais, a preservação da AVCD poderá ser usada como suprimento sangüíneo adicional ao reservatório ileal. Isto porque será grande a probabilidade de que algumas divisões vasculares adicionais no mesentério do íleo terminal serão necessárias para seu alongamento, o que poderá pôr em risco sua viabilidade.

Considerando-se a facilidade do procedimento de preservação da arcada, apesar de monótono e poder durar até uma hora, a recomendação atual do Grupo de Coloproctologia da UNICAMP é para que a arcada seja sistematicamente preservada em todos os pacientes, mesmo naqueles com mesentério favorável ou nos eleitos para anastomoses ileorretais, com ou sem reservatório ileal.

A experiência clínica deixou clara a importância dessa via alternativa de perfusão ao íleo terminal, uma vez que, mesmo após a realização de manobras de alongamento mesenterial de risco, o aporte sangüíneo manteve-se suficiente para preservar a viabilidade do reservatório ileal abaixado, sem tensão no nível do canal anal, na grande maioria dos casos. 
Em conclusão, apesar das limitações metodológicas, foi possível identificar tendência para menor ocorrência de complicações pós-operatórias, decorrentes da má-perfusão sangüínea ao reservatório, no grupo em que foi preservada a AVCD. Tendo em vista a necessidade do abaixamento do reservatório ileal ao nível da linha pectínea, isto poderá implicar a realização de ligaduras vasculares do mesentério ileal. Neste estudo, ficou evidente que a manutenção da AVCD funciona como suplemento da perfusão sangüínea ao reservatório ileal e, com isso, auxilia a preservação de sua viabilidade e funcionalidade.

\begin{abstract}
Background: The aim of this study was to analyze the efficacy of the preserved vascular arcade of the right colon (MVA) on maintaining blood perfusion to the pouch following mesenteric lenghtening maneuvers. Methods: Forty six patients submitted to total proctocolectomy and ileal pouch-anal anastomosis for the treatment of ulcerative colitis (UC) and adenomatous familial polyposis (AFP), from January 1990 to July 2000, were divided in two groups: Group With Preservation of the Arcade (WPA), 27 patients, mean age 34,0 (19-53) years, 15 (55,6\%) were female, and 24 (88,9\%) were white; and Group Without Preservation of the Arcade (WOPA), 19 patients, mean age 41,5 (13-62) years, 8 were female (42,1\%), and 18 (94,8\%) were white. Thirty patients $(65,2 \%)$ had UC and 16 (34,7\%) had AFP. Analyzes were made by the comparison of the incidence of complications due to blood perfusion failure or overtension in the ileoanal anastomosis, as well as the need of revision operation. Results: In the group WPA, 4 patients (14.8\%) had complications being related to ischemia or anastomosis overtension. In the group WOPA, it was seen in 7 patients (36,9\%). Three patients $(11,1 \%)$ of the Group WPA were submitted on to revision operation due to complications related to ischemia or anastomotic overtension and $5(26,3 \%)$ in the Group WOPA, for the same reasons. Conclusions: Besides methodological limitations, it was possible to identify a tendency to less occurrence of ischemia or anastomotic overtension related complications in the Group WPA. To perform a ileal pouch pull through operation, placing the anastomosis at the level of the pectinate line, the preserved MVA is an important technical aspect, helping to maintaining the viability and function of the pouch.
\end{abstract}

Key Words: Ulcerative colitis; Familial adenomatous polyposis; Ileal pouch; Ileoanal anastomosis.

\section{REFERÊNCIAS}

1. Goligher JC. Ulcerative colitis. In: Goligher JC. Surgery of the anus, rectum and colon. $3^{\text {rd }}$. ed., London, Baillière Tindall, 1975.

2. Parks AG, Nicholls RJ, Belliveau P. Proctocolectomy with ileal reservoir and anal anastomosis. Br J Surg 1980;67:533-538.

3. Góes JRN. Reoperações nos reservatórios ileais pélvicos. In: Habr-Gama A. Doença Inflamatória Intestinal, São Paulo, Atheneu, 1997.

4. Góes JRN. Ileal pouch-anal anastomosis: Double stapled anastomosis or mucosectomy with handsewn endoanal anastomosis? In: Reis Neto, JA. New Trends in Coloproctology, Rio de Janeiro, Revinter, 2000.

5. Góes JRN, Medeiros RR, Fagundes JJ. Colectomia total, proctomucosectomia e anastomose íleo-anal com reservatório ileal. Rev bras Colo-Proct. 1984; 4:138139.

6. Góes JRN, Fagundes JJ, Costa AM, et al. Colectomia total, proctomucosectomia, reservatório ileal em tripla alça e anastomose íleo-anal. Rev Col Bras Cir 1986;13:173-180.

7. Kiss DR, Vilarino TC, Almeida MG. Tratamento cirúrgico da retocolite ulcerativa e da polipose cólica familiar pela proctocolectomia com anastomose de reservatório ileal em $\mathbf{J}$ ao canal anal, com e sem mucosectomia retal. Rev bras de Colo-Proct 1991;11:131-141.
8. Habr-Gama A, Teixeira MG, Brunetti-Netto C et al. Restorative proctocolectomy with ileo-anal $\mathrm{J}$ pouch anastomosis for treatment of ulcerative colitis. ABCD Arq Bras Cir Dig 1992;7:72-78.

9. Góes JRN, Fagundes JJ, Coy CSR et al. Two chamber ileal pouch: an alternative design. Dis Colon Rectum 1993;36:403-404.

10. Kiss DR, Almeida MG, Baraviera AC. Bolsostomia temporária: recurso técnico para preservação de bolsas ileais pélvicas nas complicações sépticas das proctocolectomias com preservação esfincteriana. Rev bras Colo-Proct 1996;16:5-7.

11. Teixeira MG, Habr-Gama A, Rocha MES et al. Qualidade de vida dos doentes com doença inflamatória intestinal antes e após tratamento cirúrgico. Rev Bras ColoProct 1996;16:186-191.

12. Góes JRN, Fagundes JJ, Coy CSR et al. Reservatórios pélvicos. Reconstrução e reanastomose de reservatório ileal pós insucesso na primeira cirurgia. Rev Bras Colo-Proct. 1997; 7:117-121.

13. Habr-Gama A, Teixeira MG. Tratamento cirúrgico da retocolite ulcerativa - Proctocolectomia restauradora com bolsa ileal. In: Habr-Gama A. Doença Inflamatória Intestinal, São Paulo, Atheneu, 1997.

14. Góes JRN, Fagundes JJ, Coy CSR et al. Retocolectomia total e anastomose íleo-anal com reservatório ileal - Experiência de 16 anos. Rev Col Bras Cir 2000; 27:33-40.

15. Formiga GJS, Silva JH, Alves Filho EF et al. Bolsa ileal com anastomose ao canal anal: avaliação da indica- 
ção, técnica e resultados. Rev Bras Colo-Proct, 2000;20:223-226.

16. Utsunomiya J, Iwama $\mathrm{T}$, Imajo $\mathrm{M}$ et al. Total colectomy, mucosal proctectomy, and ileoanal anastomosis. Dis Colon Rectum 1980;23:459-466.

17. Smith L, Friend WG, Medweel SJ. The superior mesenteric artery. The critical factor in the pouch pull-through procedure. Dis Colon Rectum 1984;27:741-744.

18. Burnstein MJ, Schoetz DJ, Coller JA et al. Technique of mesenteric lengthening in ileal reservoir-anal anastomosis. Dis Colon Rectum 1987;30:863-866.

19. Cherqui D, Valleur P, Perniceni $T$ et al. Inferior reach of ileal reservoir in ileoanal anastomosis: experimental anatomic and angiographic study. Dis Colon Rectum 1987;30:365-371

20. Pemberton JH, Kelly KA, Beart RW Jr. et al. Ileal pouchanal anastomosis for chronic ulcerative colitis. Ann Surg 1987;206:504-513.

21. Góes JRN, Fagundes JJ, Coy CSR et al. O emprego da artéria cólica média e da arcada vascular do cólon direito na irrigação de reservatórios ileais. Rev Bras ColoProct 1994;14:169-171.

22. Góes R, Nguyen P, Huang D et al. Lengthening of the mesentery using the marginal vascular arcade of the right colon as the blood supply to the ileal pouch. Dis Colon Rectum 1995;38:893-895.

23. Thrilby RC. Optimizing results and techniques of mesentery lengthening in ileal pouch-anal anastomosis. Am J Surg 1995;169: 499-502.

24. Góes R, Beart RW, Jr. Evolution of mesentery lengthening during ileal pouch-anal anastomosis. Techniques in Coloproctology 1997;5:25-29.

25. Machado MM, Fagundes JJ, Góes JRN et al. A importância da terminação vascular íleo-ceco-cólica na confecção de reservatórios ileais com preservação da arcada vascular do cólon direito. Estudo experimental em cadáveres. Rev Bras Colo-Proct 1998;18:242-244.

26. Fegiz G, Bezzi M, Valabrega S et al. L'íleo retto-anastomosi meccanica molto bassa con reservoir ileale nel trattamento chirurgico della RCU e della poliposi diffusa del grosso intestino. Min Chir 1986; 41:453-458.

27. Heald RJ, Allen DR. Stapled ileoanal anastomosis: a technique to avoid mucosal proctectomy in the ileal pouch operation. Br J Surg 1986;73:571-572.

28. Johnston D, Holdsworth PJ, Nasmyth DG et al. Preservation of the entire anal canal in consecutive proctocolectomy for ulcerative colitis: a pilot study comparing end-to-end ileo-anal anastomosis without mucosal resection with mucosal proctectomy and endo-anal anastomosis. Br J Surg 1987;74:940-944.

29. Kmiot WA, Keighley MRB. Totally stapled abdominal restorative proctocolectomy. Br J Surg 1989;76:961-964.
30. Fazio VW, Ziv Y, Church JM et al. Ileal pouch-anal anastomoses complications and function in 1005 patients. Ann Surg 1995;222:120-127.

31. Nicholls J, Pescatori M, Motson RW et al. Restorative proctocolectomy with a three-loop ileal reservoir for ulcerative colitis and familial adenomatous polyposis. Ann Surg 1984;199:383-388.

32. Keighley MRB, Grobler S, Bain I. An audit of restorative proctocolectomy. Gut 1993;34:680-684.

33. Marcello PW, Roberts PL, Schoetz DJ et al. Long-term results of the ileoanal procedure. Arch Surg 1993;128:500-504.

Endereço para correspondência:

Juvenal Ricardo Navarro Góes

Rua Antonio Augusto de Almeida, 37

Cidade Universitária

13084-070 — Campinas-SP 